\title{
On the dynamics of workforce-mix in a manpower system
}

\author{
VU Ekhosuehi*
}

Received: 16 March 2015; Revised: 5 March 2016; Accepted: 19 October 2016

\begin{abstract}
This paper focuses on a manpower system with a fixed number of jobs that uses both permanent and temporary staff. The dynamics of workforce-mix in such a system is modelled as an optimal control problem. The objective is to find the most economical workforce-mix for the manpower system, subject to the fluctuations in workforce caused by wastage and the hiring of temporary staff. The fluctuations in the workforce-mix are modelled using a model similar to the Vidale-Wolfe advertising model. The solution is found by applying Pontryagin's principle, and a number of resulting propositions are presented along with their proofs. A real-life manpower setting is used to illustrate the utility of the model.
\end{abstract}

Key words: Manpower system, optimal control problem, Pontryagin's principle, Vidale-Wolfe model, workforce.

\section{Introduction}

The subject of workforce-mix has received considerable attention in the literature (for example, Baker [3, 4], Emmons \& Fuh [12]). Earlier studies have shown that organisations use both full-time (permanent) staff and part-time (temporary) staff to satisfy given manpower requirements. The part-time workers are hired for a finite time horizon. Universities hire part-time workers as contract staff, adjunct staff or associate staff to fill a shortfall in manpower needs. This can happen even when the institution is undergoing accreditation, and can be worsened by the loss of permanent staff, which may be due to retrenchment, retirement, death, resignation, etc. Early research has shown that the loss of staff in a manpower system is a fundamental part of the system [5]. The loss of permanent staff provides opportunities to hire temporary staff. In actual practice, the number of temporary staff hired does not exceed the shortfall in manpower requirement. This paper considers

\footnotetext{
${ }^{*}$ Department of Mathematics, University of Benin, Benin City, Nigeria, email: virtue.ekhosuehi@ uniben.edu
} 
the effect of loss of permanent staff in the dynamics of workforce-mix. This effect was not a major consideration in the works of Baker [3, 4], Emmons \& Fuh [12].

In this study, the term 'wastage' is used to refer to the loss of manpower for whatever reason. The objective is to find the most economical workforce-mix to satisfy a fixed size manpower requirement subject to the fluctuations in workforce. That is, the optimal number of temporary staff required to fill manpower shortages arising from the loss of permanent staff, which simultaneously minimises the total personnel cost. Since organisations are interested in minimising the total personnel cost, cost-effective workforce planning is essential [44]. More so, labour unions may have considerable influence on the personnel cost. In the Nigerian university system with which the author is acquainted, the allowance for excess workload is influenced by the Academic Staff Union of Universities (ASUU). This study provides bounds on the relative size of temporary staff. A model is derived for the most economical workforce-mix for a manpower system using optimal control theory within the framework of the Vidale-Wolfe advertising model [34, 42]. The model is an aggregate planning tool which is designed to assist manpower planners in finding the optimal workforce-mix for manpower systems.

From the literature, there are no examples of similar studies on manpower systems where the Vidale-Wolfe model was employed. Baker [4] had earlier considered a similar problem using a discrete-time formulation and the objective was to minimise the number of parttime staff so as to avoid excess staffing. The research expanded upon in this direction is discussed in this paper. More specifically, this study provides an alternative approach to the discrete-time formulation. The alternative approach utilises a continuous-time optimal control model. The method of least squares [25] is employed to estimate the parameters for the continuous-time model from a discrete-time dataset.

\subsection{Justification for the use of Vidale-Wolfe model in the context of manpower systems}

In Baker [3, 4] and Emmons \& Fuh [12], discrete-time models were used to schedule the workforce. For many practical purposes this is adequate. However, there are instances where the analysis could be taken further. Perhaps the most perturbing issues in manpower systems are wastage and the hiring of temporary staff to fill the shortfall in manpower requirement. Considering the possibility of wastage in a manpower system, the question arises: how does a person represent the changes in workforce as wastage can occur at virtually any time? For this a continuous-time theoretical base is necessary and so it is reasonable to represent changes in workforce as a derivative of the workforce with respect to time. It is the aim of this paper to provide a continuous-time formulation for the dynamics of workforce-mix in a manpower system and to show how it can be implemented.

The effect of a variable on another variable is commonly modelled as an interaction between the two variables [2]. This consideration leads to the hypothesis that the response of a manpower system to the shortfall in manpower may be described as an interaction between the variable representing such a response and the shortfall. Bartholomew et al. [5] reported that the loss from a group of people in an organisation is approximately proportional to the stock of people in the organisation. Apparently, wastage has a negative effect on the 
system and the hiring of temporary staff has a positive effect. This scenario is similar to the sales response to advertising in the marketing literature, where changes in the rate of sales of a product depend on two effects; the response to advertising which acts on the unsold portion of the market, and the loss due to forgetting which acts on the sold portion of the market [34]. The Vidale-Wolfe model [42] is one of the earliest management science applications to describe this phenomenon. The Vidale-Wolfe model is assumed in the context of manpower system because the model has capacity to capture losses and the interaction between two decision variables in a simplifying manner. Wastage is treated as the loss due to forgetting and the hiring of temporary staff as the advertising effort. A real-life example which is consistent with this theoretical foundation is the university system described in [31], where Osagiede et al. [31] observed that wastage and shortages in manpower needs were key issues in the system and then determine the financial implications for the university management to deal with these issues by outsourcing staff on a part-time basis. Other examples where this assumption may hold are in organisations such as restaurants and hospitals [12], labour-intensive service-oriented manpower systems [44], the iron and steel industry, automobile assembly firms, glass factories and the systems described by Baker [3, 4].

\subsection{The use of optimal control theory in the manpower context}

Wastage is outside the direct control of management of an organisation to a large extent. The management, however, exerts control over the number of temporary staff. These considerations coupled with the objective of minimising the total manpower cost imply that the management may be interested in finding an input trajectory of temporary staff which would achieve this objective, subject to workforce-mix constraints. Consequently, a controllable manpower system, which can be steered from any given state to an optimal state, is required. The central idea to model such a system is optimal control theory [43]. The use of optimal control in finding an optimal workforce size has been analysed elsewhere in the literature $[10,16]$. These studies used discrete-time formulations to obtain optimal strategies for the manpower system. Even so, the optimal solutions were not in closed form. As mentioned earlier, wastage may occur at any instant of time. Thus a continuous-time optimal control model is employed for the manpower system to obtain closed form expressions for the optimal solutions.

In a continuous-time optimal control model, there are two classes of variables: the state variables and the control variables. In this study, the state variable is defined as the fraction of permanent staff at a certain moment and the control variable as the fraction of temporary staff at a certain moment. The simplest control problem [21] deals with the selection of a continuous control function, $u(t), t_{0} \leq t \leq T$, to

$$
\max _{u(t)} \int_{t_{0}}^{T} f(t, x(t), u(t)) \mathrm{d} t
$$

subject to

$$
\frac{\mathrm{d} x(t)}{\mathrm{d} t}=g(t, x(t), u(t)),
$$

where $x\left(t_{0}\right)=x_{0}$ is fixed and $x(T)$ is free. In equations (1) and (2), $f$ and $g$ are assumed to be continuously differentiable functions of the three independent arguments, none of 
which is a derivative, and $x(t)$ is a state variable at moment $t$. The state variable $x(t)$ changes over time according to the differential equation (2). The control $u(t)$ influences the objective (1) both directly through its own value and indirectly through its impact on the evolution of the state variable $x(t)$. The state equation (2), which is also called the state-transition equation, is a first-order differential equation. The necessary conditions of optimality to the control problem may be derived from the Pontryagin's principle and Green's theorem [2, 21, 34].

\section{Related work}

Manpower planning involves strategies aimed at providing a continuous matching of available personnel with certain qualifications to the manpower requirements of the system [41]. Agnihothri et al. [1] studied a workforce-mix problem for two heterogeneous job types of a system in steady-state with the objective of minimising the sum of the average service costs and the customer delay costs per unit time. Bordoloi [6] derived steady-state workforce levels for the knowledge-mix within a firm with a view to minimising total labour. Zhu \& Sherali [44] considered workforce fluctuations based on an expected demand profile. Lee \& Vairaktarakis [24] addressed a workforce planning problem of serial assembly lines by minimising the minimum workforce requirements over all production cycles. Near-optimal solutions were obtained for the problem using several heuristics. Corominas et al. [9] developed a discrete-time workforce planning model which integrates production and cash management decisions for a manpower system. Harper et al. [18] suggested that it is cost beneficial to increase the number of permanently employed staff to account for fluctuations in demand in a manpower system.

Edwards [11] reviewed the models developed in the manpower planning literature and concluded that the ease of use of models is more important than the theoretical sophistication. Stanford [35] considered the system maintenance cost and the individual member earnings as the cost incurred in a manpower system. Rao [33] developed a dynamic programming model for a manpower system with the objective of minimising the manpower system costs. The model in this paper is different from Rao's model in that our model is an optimal control model in continuous-time. Flynn [14] studied the flows in a multigrade manpower system using a deterministic dynamic programming model. Chu \& Lin [7] developed a non-linear model for a manpower allocation problem with an interdependent relationship between the assignment of staff and the average travel time. Hegde \& Tadikamalla [20] applied mathematical programming techniques to personnel scheduling in a manpower system.

The mixed exponential distribution [26], the lognormal distribution [8], the non-parametric competing risk model [28] and bootstrap techniques [23] have all found applications in manpower planning. The manpower system has also been described using the entropy approach. McClean \& Abodunde [27] and Vassiliou [38] employed the Shannon-type entropy as a measure of the degree of experience in a manpower system. Tyler [36] presented a model of manpower systems based on the concept of thermodynamics. The model utilised the Boltzmann entropy.

Guerry [17], Kipouridis \& Tsaklidis [22] and Vassiliou \& Tsantas [40] analysed graded 
manpower systems using discrete-time Markov chains. The semi-Markov models [37, 39] and the continuous-time Markov models [15, 29] have also been used to describe graded manpower systems. More details on the use of Markov models for manpower planning may be found in $[5,32]$.

The use of continuous-time Markov models for graded manpower systems is a source of inspiration to develop a continuous-time optimal control model for manpower systems. This consideration is an addition to the existing literature on the subject of workforce-mix as results are in a closed form, unlike the discrete-time formulations [3, 4, 12, 33]. More so, the representation of the changes in workforce as a state-transition model which depends on loss of permanent staff and an organisation's response to hiring temporary employees which acts on the manpower shortage experienced by the organisation is novel.

\section{Theoretical framework}

This section contains the formulation of the problem and the solution. An asterisk $\left(^{*}\right)$ is added to a variable to represent the optimal value and the hat symbol $\left({ }^{\wedge}\right)$ on a parameter is used to denote an estimate. The routines for implementing the solutions and the schematic displays are carried out in the MATLAB environment. It is important to note that the use of the term 'temporary staff' in this study is synonymous to 'part-time staff', 'contract staff', 'casual staff' or 'outsourced staff'.

\subsection{Model set-up}

This study assumes a fixed size manpower requirement for a manpower system that uses both permanent and temporary staff. The permanent staff may belong to a labour union, which is capable of influencing wages through collective bargaining. It is assumed that the initial size of permanent staff is known (i.e., no 'ghost' worker). The loss of permanent staff in the system is due to wastage. The wastage rate is assumed to have a significant effect on the manpower system. The temporary staff are hired whenever under-staffing exists. It is assumed that the unit cost of hiring temporary staff is constant and that the number of temporary staff is under control. Inflation is not considered within the cost function. Another assumption is that allocation problems are not considered in the workforce-mix. This study also assumes that the fluctuations in workforce may be described by the VidaleWolfe model [13, 34, 42].

Let $N$ be a fixed workforce size for the manpower system. Let $x(t)$ denote the fraction of permanent staff at moment $t \in[0, T]$ relative to the manpower requirement (or simply, the fraction of permanent staff at moment $t$ ). The interval $[0, T]$ is a fixed time horizon. The fraction of the permanent workforce at the beginning of the period $t=0$ is denoted as $x(0)=x_{0}$. For $N x(t)<N$, the manpower system is under-staffed. The shortfall $N(1-$ $x(t)$ ) creates vacancies which are either filled by recruiting additional permanent staff or by outsourcing work to temporary staff. Let $u(t)$ represent the fraction of temporary staff at moment $t$ relative to the manpower requirement. Then the manpower cost, $C(u(t), x(t))$, is expressed as

$$
C(u(t), x(t))=c_{1} u(t)+c_{2} x^{k}(t), k \geq 1, c_{1}, c_{2}>0,
$$


where the first term is the total cost of hiring temporary staff and the second term is the total retention cost. The parameters $c_{1}$ and $c_{2}$ are constants in monetary units. The constant $k$ describes the diseconomies when scaling up workers' wages consequent upon workers' agitations for a better pay and the collective bargaining by the labour union and the management of the manpower system.

Since the fluctuations in the workforce are induced by two effects, i.e., the wastage from the system and the hiring of temporary staff to bridge the shortfall in manpower requirement, the state-transition model, analogous to the Vidale-Wolfe model, may be written as

$$
\frac{\mathrm{d} x(t)}{\mathrm{d} t}=-\gamma_{1} x(t)+\gamma_{2} u(t)(1-x(t))
$$

where $\gamma_{1} \geq 0$ is the attrition effect parameter and $\gamma_{2}>0$ is the reaction effect parameter. The constant $\gamma_{1}$ may be thought of as the difference between the wastage rate and the recruitment rate in such fashion that the latter does not exceed the former. The term $-\gamma_{1} x(t)$ describes the reduction in workforce due to wastage, while the term $\gamma_{2} u(t)(1-x(t))$ captures the hiring of temporary staff to bridge the shortfall in the manpower requirement. Technically, the workforce-mix problem considered in this study is stated as follows.

Determine the fraction of temporary staff $u(t)$ and the permanent staff $x(t)$ for the period $0 \leq t \leq T$ to

$$
\min _{u(t)} \int_{0}^{T}\left(c_{1} u(t)+c_{2} x^{k}(t)\right) \mathrm{d} t
$$

subject to

$$
\frac{\mathrm{d} x(t)}{\mathrm{d} t}=-\gamma_{1} x(t)+\gamma_{2} u(t)(1-x(t)),
$$

where $0<u(t) \leq(1-x(t))$ for $x(t)<1$ and $u(t)=0$ otherwise. Also, $x(0)=x_{0}, t \in[0, T]$. The bounds for the control imply that the number of temporary staff does not exceed the shortfall in the manpower requirement. The model set-up for the workforce-mix problem is therefore a bounded optimal control problem with the bounds being the closed interval $[0,1-x(t)]$.

\subsection{Model solution}

The Pontryagin's principle is applied to the bounded optimal control problem. The Hamiltonian, $H$, with arguments $(x(t), u(t), \lambda(t))$, is

$$
H=c_{1} u(t)+c_{2} x^{k}(t)+\lambda(t)\left(-\gamma_{1} x(t)+\gamma_{2} u(t)(1-x(t))\right),
$$

where $\lambda(t)$ is a multiplier function, which defines the marginal valuation of the permanent staff at moment $t$. The influence equation is obtained as

$$
\frac{\mathrm{d} \lambda(t)}{\mathrm{d} t}=-\frac{\partial H}{\partial x(t)}=-\left(k c_{2} x^{k-1}(t)+\lambda(t)\left(-\gamma_{1}-\gamma_{2} u(t)\right)\right)
$$

The Lagrangian function, $L$, for the Hamiltonian subject to the control bounds is

$$
L=c_{1} u(t)+c_{2} x^{k}(t)+\lambda(t)\left(-\gamma_{1} x(t)+\gamma_{2} u(t)(1-x(t))\right)+\rho_{1} u(t)+\rho_{2}(1-x(t)-u(t)),
$$


where $\rho_{1}$ and $\rho_{2}$ are the Lagrangian multipliers. The necessary conditions for $u(t)$ to minimise the bounded optimal control problem are

$$
\frac{\partial L}{\partial u(t)}=c_{1}+\gamma_{2} \lambda(t)(1-x(t))+\rho_{1}-\rho_{2}=0,
$$

where $\rho_{1} \geq 0, \rho_{1} u(t)=0, \rho_{2} \geq 0$ and $\rho_{2}(1-x(t)-u(t))=0$. Thus, the following cases arise.

\subsubsection{Case 1}

Suppose $\rho_{1}=\rho_{2}=0$. Then $\lambda(t)=-\frac{c_{1}}{\gamma_{2}(1-x(t))}$, so that

$$
\frac{\mathrm{d} \lambda(t)}{\mathrm{d} t}=-\frac{c_{1}}{(1-x(t))^{2}} \frac{d x(t)}{d t} .
$$

The influence equation (7) becomes

$$
\frac{c_{1}}{(1-x(t))^{2}} \frac{\mathrm{d} x(t)}{\mathrm{d} t}=k c_{2} x^{k-1}(t)+\frac{c_{1}}{\gamma_{2}(1-x(t))}\left(\gamma_{1}+\gamma_{2} u(t)\right) .
$$

Making a substitution for $u(t)$ from the state-transition equation (4) into equation (9) yields

$$
x^{k+1}(t)-2 x^{k}(t)+x^{k-1}(t)+\frac{c_{1} \gamma_{1}}{2 c_{2} \gamma_{2}}=0 .
$$

When $k=1$, no real solution exists, unless $\gamma_{1}=0$. This is because

$$
4\left(1+\frac{c_{1} \gamma_{1}}{2 c_{2} \gamma_{2}}\right)>4
$$

for $\gamma_{1} \neq 0$. Similarly, when $k=2$, no real solution exists, unless $\gamma_{1}=0$. To see this, let $x(t)=\varphi \cos \theta+\frac{2}{3}, \varphi>0$. Then equation (10) becomes

$$
\varphi^{3} \cos ^{3} \theta-\frac{1}{3} \varphi \cos \theta+\left(\frac{c_{1} \gamma_{1}}{2 c_{2} \gamma_{2}}+\frac{2}{27}\right)=0 .
$$

By comparing equation (11) with the trigonometric identity $4 \cos ^{3} \theta-3 \cos \theta=\cos 3 \theta$, $\varphi=\frac{2}{3}$ and

$$
\theta=\frac{1}{3}\left(2 n \pi+\cos ^{-1}\left(-\left(1+\frac{27 c_{1} \gamma_{1}}{4 c_{2} \gamma_{2}}\right)\right)\right), \quad n=0,1,2 .
$$

Therefore, the optimal solution becomes

$$
x^{*}(t)=\frac{2}{3}\left(1+\cos \left(\frac{2 n \pi}{3}+\frac{1}{3} \cos ^{-1}\left(-\left(1+\frac{27 c_{1} \gamma_{1}}{4 c_{2} \gamma_{2}}\right)\right)\right)\right), \quad n=0,1,2 .
$$

There are three solutions of $x^{*}(t)$, for $n=0,1,2$, in equation (12), which are independent of time. It is easily seen that the stationary solutions are not feasible, unless $\gamma_{1}=0$. This is because the term $\cos ^{-1}\left(-\left(1+\frac{27 c_{1} \gamma_{1}}{4 c_{2} \gamma_{2}}\right)\right)$ cannot exist as a real-value for $\gamma_{1} \neq 0$. 
In particular, for $\gamma_{1} \neq 0,1+\frac{27 c_{1} \gamma_{1}}{4 c_{2} \gamma_{2}}$ exceeds the bounds of the cosine function, which oscillates between the values \pm 1 .

For $k \neq 1,2$, numerical techniques are applied. Using Newton's method yields

$$
x_{(i+1)}(t)=x_{(i)}(t)-\frac{f_{(i)}}{f_{(i)}^{\prime}},
$$

where

$$
\begin{aligned}
& f_{(i)}=x_{(i)}^{k+1}(t)-2 x_{(i)}^{k}(t)+x_{(i)}^{k-1}(t)+\frac{c_{1} \gamma_{1}}{2 c_{2} \gamma_{2}}, \\
& f_{(i)}^{\prime}=(k+1) x_{(i)}^{k}(t)-2 k x_{(i)}^{k-1}(t)+(k-1) x_{(i)}^{k-2}(t),
\end{aligned}
$$

and $i=0,1,2, \ldots$, is the number of iterations. This solution is independent of time, so that $x_{(i+1)}(t)$ may be written as $x_{(i+1)}$. In essence, the optimal solution, say $x_{(i+1)}=x^{*}$, is independent of time and the control, $u^{*}$, is also independent of time. Thus,

$$
u^{*}=\frac{\gamma_{1} x^{*}}{\gamma_{2}\left(1-x^{*}\right)}
$$

and $\frac{\mathrm{d} x(t)}{\mathrm{d} t}=0$.

\subsubsection{Case 2}

Suppose $\rho_{1}=0, \rho_{2}>0$ and $\gamma_{1}>0$. Then $\lambda(t)>-\frac{c_{1}}{\gamma_{2}(1-x(t))}$ and the control is at the upper bound. In this case, the state-transition equation is a non-linear autonomous system. The analytic solution $x(t)$ which satisfies the initial condition $x(0)=x_{0}$ is obtained using the transformation $z=(1-x(t))^{-1}$ so that the state-transition equation becomes

$$
\frac{\mathrm{d} z}{\mathrm{~d} t}=-\gamma_{1}\left(z^{2}-z-\frac{\gamma_{2}}{\gamma_{1}}\right)
$$

This leads to the evaluation of the integrals

$$
\frac{1}{\alpha_{1}-\alpha_{2}} \int\left(\frac{1}{z-\alpha_{1}}-\frac{1}{z-\alpha_{2}}\right) \mathrm{d} z=-\gamma_{1} \int \mathrm{d} t,
$$

where

$$
\alpha_{1}=\frac{1}{2}\left(1+\left(1+4 \frac{\gamma_{2}}{\gamma_{1}}\right)^{1 / 2}\right)>0
$$

and

$$
\alpha_{2}=\frac{1}{2}\left(1-\left(1+4 \frac{\gamma_{2}}{\gamma_{1}}\right)^{1 / 2}\right)<0 .
$$

After some simplifications, the optimal fraction of permanent staff is obtained as

$$
x^{*}(t)=1-\frac{1-\phi \exp \left(-\left(\alpha_{1}-\alpha_{2}\right) \gamma_{1} t\right)}{\alpha_{1}-\alpha_{2} \phi \exp \left(-\left(\alpha_{1}-\alpha_{2}\right) \gamma_{1} t\right)},
$$


where

$$
\phi=\frac{1-\alpha_{1}\left(1-x_{0}\right)}{1-\alpha_{2}\left(1-x_{0}\right)}
$$

and

$$
\alpha_{1}-\alpha_{2}=\left(1+4 \frac{\gamma_{2}}{\gamma_{1}}\right)^{1 / 2}
$$

\subsubsection{Case 3}

Suppose $\rho_{1}>0, \rho_{2}=0$ and $\gamma_{1}>0$. Then $\lambda(t)<-\frac{c_{1}}{\gamma_{2}(1-x(t))}$ and $u^{*}(t)=0$. Thus the state-transition equation becomes a linear autonomous system with the solution

$$
x^{*}(t)=x_{0} \exp \left(-\gamma_{1} t\right)
$$

This solution holds for $x^{*}(t) \geq 1$. This is because $u^{*}(t)=0$ implies no under-staffing.

\subsubsection{Case 4}

Suppose $\rho_{1}, \rho_{2}>0$. Then $\rho_{1} u(t)=0$ implies that $u^{*}(t)=0$. Similarly, $\rho_{2}(1-x(t)-u(t))=$ 0 implies that $1-x^{*}(t)-u^{*}(t)=0$. Since $u^{*}(t)=0, x^{*}(t)=1$. This case also excludes under-staffing.

\subsection{Some useful insights}

Some insights may be drawn from the solution to the workforce-mix problem. These insights are presented as propositions below.

Proposition 1: Whenever excess staffing exists and the optimal workforce size is independent of time, then the attrition effect is zero.

Proof: Suppose $x^{*}(t)$ is below 1. Then it can never exceed 1 again. This is because, for $x^{*}(t)=1$ in equation (4), its derivative is negative. Thus, the hiring of temporary staff is required. If $x^{*}+u^{*}(t)>1$, then $u^{*}(t)=0$ as $u^{*}(t)$ cannot exceed $1-x^{*}(t)$. When the workforce size is independent of time, $\frac{\mathrm{d} x(t)}{\mathrm{d} t}=0$. From the state-transition equation therefore, $\gamma_{1}=0$.

Proposition 2: Whenever $0 \leq \phi^{2} \exp \left(-2\left(\alpha_{1}-\alpha_{2}\right) \gamma_{1} t\right)<1, x^{*}(t)$ is below 1 .

Proof: Clearly $\phi^{2} \exp \left(-2\left(\alpha_{1}-\alpha_{2}\right) \gamma_{1} t\right) \geq 0$ as $\phi^{2} \geq 0$ and $\exp \left(-2\left(\alpha_{1}-\alpha_{2}\right) \gamma_{1} t\right) \geq 0$. To show that $0 \leq \phi^{2} \exp \left(-2\left(\alpha_{1}-\alpha_{2}\right) \gamma_{1} t\right)<1$ implies $x^{*}(t)$ is below 1 , it is sufficient to prove that

$$
\frac{1-\phi \exp \left(-\left(\alpha_{1}-\alpha_{2}\right) \gamma_{1} t\right)}{\alpha_{1}-\alpha_{2} \phi \exp \left(-\left(\alpha_{1}-\alpha_{2}\right) \gamma_{1} t\right)}>0
$$

whenever $0 \leq \phi^{2} \exp \left(-2\left(\alpha_{1}-\alpha_{2}\right) \gamma_{1} t\right)<1$. Let $\omega=\left(\alpha_{1}-\alpha_{2}\right) \gamma_{1}$. If $0 \leq \phi^{2} \exp (-2 \omega t)<1$, 
then

$$
\begin{aligned}
\frac{1-\phi \exp (-\omega t)}{\alpha_{1}-\alpha_{2} \phi \exp (-\omega t)} & =\frac{1-\phi^{2} \exp (-2 \omega t)}{\left(\alpha_{1}-\alpha_{2} \phi \exp (-\omega t)\right)(1+\phi \exp (-\omega t))} \\
& =\frac{1-\phi^{2} \exp (-2 \omega t)}{\frac{1}{2}\left(\left(1-\phi^{2} \exp (-2 \omega t)\right)+\left(1+4 \frac{\gamma_{2}}{\gamma_{1}}\right)^{1 / 2}(1+\phi \exp (-\omega t))^{2}\right)} \\
& >0 .
\end{aligned}
$$

For $\phi \geq 0,1-\phi \exp (-\omega t)>0$ and $\alpha_{1}-\alpha_{2} \phi \exp (-\omega t)>0$. Thus,

$$
\frac{1-\phi \exp (-\omega t)}{\alpha_{1}-\alpha_{2} \phi \exp (-\omega t)}>0
$$

Consider the case when $\phi<0$. Then

$$
\begin{aligned}
\frac{1-\phi \exp (-\omega t)}{\alpha_{1}\left(1-\frac{\alpha_{2}}{\alpha_{1}} \phi \exp (-\omega t)\right)} & =\frac{1}{\alpha_{1}}(1-\phi \exp (-\omega t))\left(1-\frac{\left(-\alpha_{2}\right)}{\alpha_{1}}(-\phi) \exp (-\omega t)\right)^{-1} \\
& =\frac{1}{\alpha_{1}}(1-\phi \exp (-\omega t)) \sum_{r=0}^{\infty}\left(\frac{\left(-\alpha_{2}\right)}{\alpha_{1}}(-\phi) \exp (-\omega t)\right)^{r} \\
& >\frac{1}{\alpha_{1}}(1-\phi \exp (-\omega t))\left(1+\frac{\left(-\alpha_{2}\right)}{\alpha_{1}}(-\phi) \exp (-\omega t)\right) \\
& =\frac{1}{\alpha_{1}^{2}}(1-\phi \exp (-\omega t))\left(\alpha_{1}+\alpha_{2} \phi \exp (-\omega t)\right)
\end{aligned}
$$

and

$$
\frac{1}{2 \alpha_{1}^{2}}\left(\left(1-\phi^{2} \exp (-2 \omega t)\right)+\left(1+4 \frac{\gamma_{2}}{\gamma_{1}}\right)^{1 / 2}(1-\phi \exp (-\omega t))^{2}\right)>0
$$

It follows that

$$
1-\frac{1-\phi \exp \left(-\left(\alpha_{1}-\alpha_{2}\right) \gamma_{1} t\right)}{\alpha_{1}-\alpha_{2} \phi \exp \left(-\left(\alpha_{1}-\alpha_{2}\right) \gamma_{1} t\right)}<1
$$

Remark 1: When $\phi^{2} \exp \left(-2\left(\alpha_{1}-\alpha_{2}\right) \gamma_{1} t\right) \geq 1, x^{*}(t) \geq 1$ and $u^{*}(t)=0$.

Proposition 3: Whenever $0 \leq \phi^{2} \exp \left(-2\left(\alpha_{1}-\alpha_{2}\right) \gamma_{1} t\right)<1$, the optimal solution to the workforce-mix problem is

$$
x^{*}(t)=1-\frac{1-\phi \exp \left(-\left(\alpha_{1}-\alpha_{2}\right) \gamma_{1} t\right)}{\alpha_{1}-\alpha_{2} \phi \exp \left(-\left(\alpha_{1}-\alpha_{2}\right) \gamma_{1} t\right)},
$$

with

$$
u^{*}(t)=1-x^{*}(t)
$$

Proof: The proof follows from Case 2 of the workforce-mix problem and the proof of Proposition 2. 
Proposition 4: Given the initial state $x_{0}$, the optimal fraction of permanent staff tends to

$$
1+\frac{\gamma_{1}}{2 \gamma_{2}}\left(1-\left(1+4 \frac{\gamma_{2}}{\gamma_{1}}\right)^{1 / 2}\right)
$$

when the time period is sufficiently large.

Proof: When $T$ is large, then $\exp \left(-\left(\alpha_{1}-\alpha_{2}\right) \gamma_{1} t\right) \rightarrow 0$. This is equivalent to writing $\lim _{t \rightarrow \infty} \exp \left(-\left(\alpha_{1}-\alpha_{2}\right) \gamma_{1} t\right)=0$. It follows that $x^{*}(T) \rightarrow \lim _{t \rightarrow \infty} x^{*}(t)$ as the time period elapses. Thus,

$$
\begin{aligned}
\lim _{t \rightarrow \infty} x^{*}(t) & =1-\frac{1}{\alpha_{1}} \\
& =1-\frac{2}{1+\left(1+4 \frac{\gamma_{2}}{\gamma_{1}}\right)^{1 / 2}} \\
& =1+\frac{\gamma_{1}}{2 \gamma_{2}}\left(1-\left(1+4 \frac{\gamma_{2}}{\gamma_{1}}\right)^{1 / 2}\right) .
\end{aligned}
$$

Remark 2: The managerial implications of Proposition 4 are that: additional staff not more than $N\left|x^{*}(t)-x(t)\right|$ should be recruited at any time instant $t \in[0, T]$ whenever $x^{*}(t)<x(t)$; otherwise, they should be retrenched.

\subsection{Estimation of parameters of the transition model}

The model presented in this paper is a continuous-time model. However, data on the staff strength in a manpower system are usually available on a discrete-time basis - monthly, quarterly, bi-annually or annually. Thus, the discrete-time model may be used as a proxy for the continuous-time process. Suppose the manpower requirement, $N$, is known and historical data at specific periods, $t=1,2, \ldots, \eta$, are available on the fraction of temporary staff, $u_{t}$, and the fraction of permanent staff, $x_{t}$. Then the state-transition equation can be specified in the discrete form as

$$
x_{t}=\left(1-\gamma_{1}\right) x_{t-1}+\gamma_{2} u_{t-1}\left(1-x_{t-1}\right)+\varepsilon_{t}, \quad t=1,2,3, \ldots, \eta,
$$

where $\varepsilon_{t}$ is the error term, The parameters $\gamma_{1}$ and $\gamma_{2}$ are estimated by minimising the sum of square errors, $\sum_{t=1}^{\eta} \varepsilon_{t}^{2}$. Thus, by the method of least squares,

$$
\hat{\gamma}_{1}=1-\left[\begin{array}{ll}
1 & 0
\end{array}\right]\left(\left[\mathbf{X}_{-1} \mathbf{\Pi}\right]^{\prime}\left[\mathbf{X}_{-1} \mathbf{\Pi}\right]\right)^{-1}\left[\mathbf{X}_{-1} \mathbf{\Pi}\right]^{\prime} \mathbf{X},
$$

and

$$
\hat{\gamma}_{2}=\left[\begin{array}{ll}
0 & 1
\end{array}\right]\left(\left[\mathbf{X}_{-1} \mathbf{\Pi}\right]^{\prime}\left[\mathbf{X}_{-1} \mathbf{\Pi}\right]\right)^{-1}\left[\mathbf{X}_{-1} \mathbf{\Pi}\right]^{\prime} \mathbf{X},
$$

where $\mathbf{X}_{-1}$ is an $(\eta-1) \times 1$ vector of $x_{t-1}, \boldsymbol{\Pi}$ is an $(\eta-1) \times 1$ vector of the product $u_{t-1}\left(1-x_{t-1}\right)$ and $\mathbf{X}$ is an $(\eta-1) \times 1$ vector of $x_{t}$. 


\section{Numerical illustration}

To illustrate the use of the model in Section 3 consider the pattern of staff distribution in Table 1 of a faculty in a university system [30]. The system is designed in such a fashion that staff in the position of Graduate Assistant are entirely temporary staff. The unit cost per annum of each staff category is computed as the average salary. Thus $c_{1}=1.32$ million naira ${ }^{1}$ per annum and $c_{2}=3.87$ million naira per annum. Let the manpower requirement be the number of staff at $t=5$ so that no excess staffing is considered. At $t=0$, the fraction of permanent staff relative to the manpower requirement is computed as $x_{0}=0.8017$.

\begin{tabular}{lcccccc}
\hline $\mathrm{t}$ & 0 & 1 & 2 & 3 & 4 & 5 \\
\hline Graduate assistant & 8 & 3 & 4 & 8 & 11 & 15 \\
Assistant lecturer \& above & 93 & 101 & 100 & 98 & 98 & 101 \\
\hline
\end{tabular}

Table 1: Historical data on the workforce in a university faculty.

The parameters of the state-transition equation are estimated from Table 1 using equations (21) and (22) as $\hat{\gamma}_{1}=0.0425$ and $\hat{\gamma}_{2}=5.2336$ (see appendix). The optimal workforce-mix is solved for $x^{*}(t)+u^{*}(t) \leq N$. For $x^{*}(t)+u^{*}(t)<N$, no real solution exists for the algebraic equation $(10), k=3, \ldots, 8$. For this reason, the optimal solution in Case 2 , for which $x^{*}(t)+u^{*}(t)=N$, is employed. The values of $\alpha_{1}$ and $\alpha_{2}$ are computed as 11.6122 and -10.6122 , respectively. These results agree with the a priori specifications. The dynamics of the optimal workforce are extrapolated for a 10-year period. For $\phi^{2} \exp \left(-2\left(\alpha_{1}-\alpha_{2}\right) \gamma_{1} t\right) \geq$ $1, x^{*}(t) \geq 1$. Consequent upon this, the control $u^{*}(t)$ is set equal to zero. This is in line with the Remark 1. Using the assertion in Proposition 3, the trajectories of the optimal fraction of permanent staff to be retained and the optimal fraction of temporary staff to be hired are obtained. The results are displayed in Figure 1 and Figure 2. The MATLAB source code for the computations and graphical displays are given in the appendix. The limiting fraction of permanent staff is obtained as $\lim _{t \rightarrow \infty} x(t)=0.9139$.

Figure 1 indicates that a continuous recruitment of permanent staff is required until the fraction of permanent staff reaches the limiting value. Figure 2 shows that the hiring of temporary staff should be reduced until the fraction of temporary staff stabilizes at the value 0.0861. These results agree with the assertion in Proposition 3. Thus the optimal workforce-mix for the faculty is that academic staff in the rank of Assistant Lecturer $\&$ above should be increased to $91.39 \%$ of the manpower requirement and $8.61 \%$ of the academic staff should be recruited as Graduate Assistants. This deduction is in line with Harper et al. [18].

\section{Conclusion}

This study discussed the formulation of a continuous-time optimal control model for the workforce-mix in a manpower system. The workforce-mix is made up of temporary and

\footnotetext{
${ }^{1}$ Naira is the currency of Nigeria. As at the time of writing this paper, 1 naira is equivalent to 0.06226 South African Rand.
} 


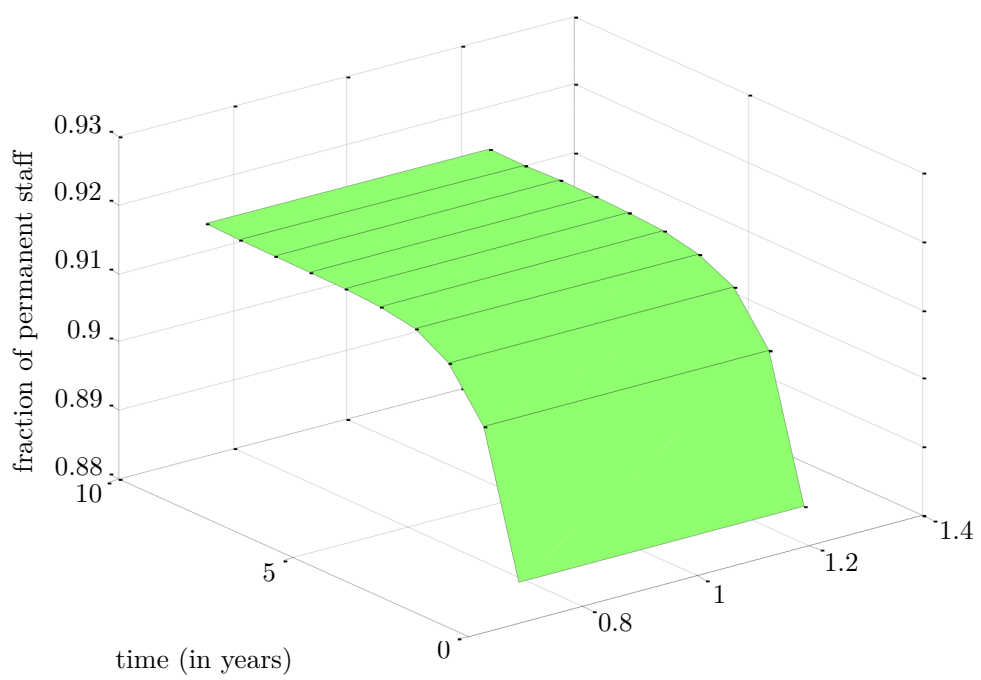

Figure 1: Dynamics of the optimal fraction of permanent staff.

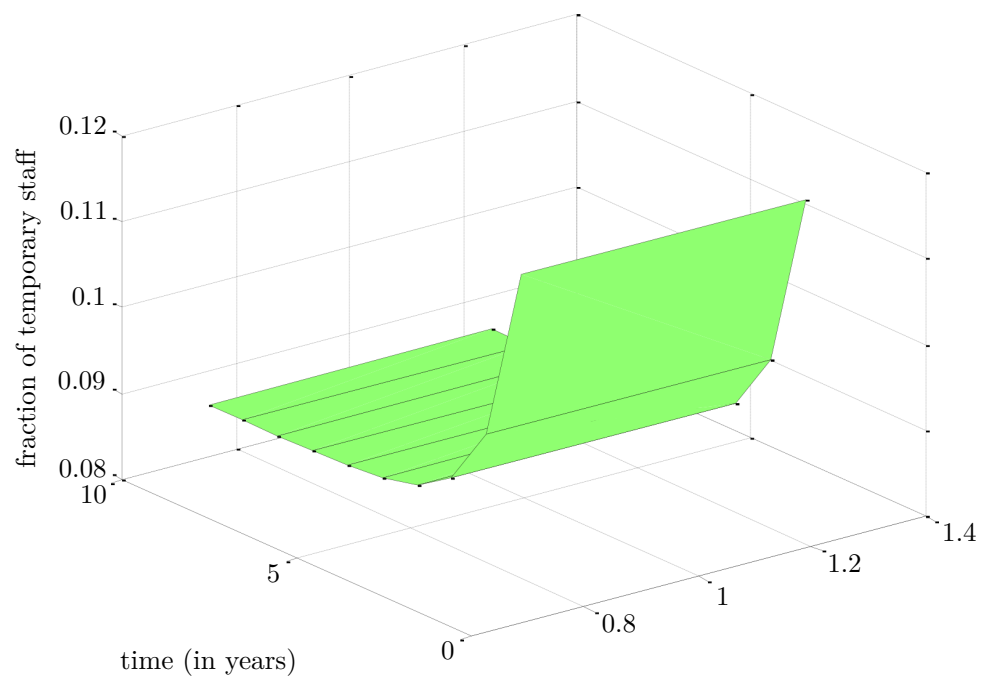

Figure 2: Dynamics of the optimal fraction of temporary staff. 
permanent staff. The scenario where wastage rate has a significant effect on the manpower system was considered. The fundamentals of optimal control theory and the Vidale-Wolfe model were employed to derive a formula for the fraction of permanent staff that should be retained as well as the fraction of temporary staff that should be hired in a manpower system. Findings reveal that: (i) the attrition effect is zero whenever excess staffing exists and the optimal workforce size is independent of time, (ii) whenever temporary staff are hired, it should be equal to the shortfall in the manpower requirement, and (iii) a steadystate value exists for the fraction of permanent staff.

The manpower model in this paper is a suitable alternative to the discrete-time models in the literature. It is worthy of note that the most rapid approach path (MRAP) as in [19] may as well be employed instead of the Pontryagin's principle since the workforce-mix problem is a linear control problem (i.e., the problem is linear in the control). Nonetheless, the same conclusions are expected. The current model is not without limitations. The assumption of a fixed manpower requirement may not hold in the long-run situation owing to variations in demand, which are important for staffing issues. The model should be used with caution as allocation problems and the learning period for hired temporary staff were not included in the model formulation. In addition, the exclusion of inflation in the cost function and the assumption of a constant unit cost of hiring temporary staff, without due considerations to the bargaining power of temporary staff and proficiency, are shortcomings to the model implementation. More so the model is deterministic. In the real-world scenario, the wastage (or attrition) rate is not entirely predictable. To refine the model, further research may be undertaken to develop a probabilistic model in continuous-time for the manpower system which may also incorporate the skill-mix of the workforce from a real-world perspective.

\section{Acknowledgement}

I acknowledge the editor and the anonymous reviewers for their helpful comments which have greatly improved the quality of the earlier manuscript.

\section{References}

[1] Agnihothri S, Mishra A \& Simmons D, 2003, Workforce cross-training decisions in field service systems with two job types, Journal of the Operational Research Society, 54(4), pp. 410-418.

[2] Anita S, Arnautu V \& Capasso V, 2011, An introduction to optimal control problems in life sciences and economics, $1^{\text {st }}$ Edition, Springer, New York.

[3] BAKER KR, 1974, Scheduling a full-time workforce to meet cyclic staffing requirements, Management Science, 20(12), pp. 1561-1568.

[4] BAKER KR, 1974, Scheduling full-time and part-time staff to meet cyclic requirements, Journal of the Operational Research Society, 25(1), pp. 65-76.

[5] Bartholomew DJ, Forbes AF \& McClean SI, 1991, Statistical techniques for manpower planning, $2^{\text {nd }}$ Edition, John Wiley \& Sons, Chichester.

[6] Bordoloi S, 2006, A control rule for recruitment planning in engineering consultancy, Journal of Productivity Analysis, 26(2), pp. 147-163. 
[7] Chu SC \& Lin CK, 1993, A manpower allocation model of job specialization, Journal of the Operational Research Society, 44(10), pp. 983-989.

[8] Chu SC \& LIN CK, 1994, Cohort analysis technique for long-term manpower planning: The case of a Hong Kong tertiary institution, Journal of the Operational Research Society, 45(6), pp. 696-709.

[9] Corominas A, Lusa A \& Olivella J, 2012, A detailed workforce planning model including nonlinear dependence of capacity on the size of the staff and cash management, European Journal of Operational Research, 216(2), pp. 445-458.

[10] CuRTis RG, 1987, Top management systems for forecasting and monitoring pay costs and staff numbers, Journal of the Operational Research Society, 38(10), pp. 935-940.

[11] Edwards JS, 1983, A survey of manpower planning models and their application, Journal of the Operational Research Society, 34(11), pp. 1031-1040.

[12] Emmons H \& Fun DS, 1997, Sizing and scheduling a full-time and part-time workforce with off-day and off-weekend constraints, Annals of Operations Research, 70(1), pp. 473-492.

[13] Feichtinger G, Hartl RF \& Sethi SP, 1994, Dynamic optimal control models in advertising: recent developments, Management Science, 40(2), pp. 195-226.

[14] Flynn J, 1981, A dynamic programming model of fractional flows with application to maintenance and replacement problems, Mathematics of Operations Research, 6(3), pp. 405-419.

[15] Gerontidis II, 1990, On certain aspects of non-homogeneous markov systems in continuous time, Journal of Applied Probability, 27(3), pp. 530-544.

[16] Grinold RC \& Stanford RE, 1974, Optimal control of a graded manpower system, Management Science, 20(8), pp. 1201-1216.

[17] Guerry M, 1997, Properties of calculated predictions of grade sizes and the associated integer valued vectors, Journal of Applied Probability, 34(1), pp. 94-100.

[18] Harper PR, Powell N \& Williams JE, 2010, Modelling the size and skill-mix of hospital nursing teams, Journal of the Operational Research Society, 61(5), pp. 768-779.

[19] Hartl RF \& Feichtinger G, 1987, A new sufficient condition for most rapid approach paths, Journal of Optimization Theory and Applications, 54(2), pp. 403-411.

[20] Hegde G \& Tadikamalla PR, 1990, Interactive workforce management on a PC: An industryuniversity partnership, European Journal of Operational Research, 48(2), pp. 275-280.

[21] Kamien M \& Schwartz N, 1992, Dynamic optimization: the calculus of variation and optimal control in economics and management, $2^{\text {nd }}$ Edition, Elsevier, Amsterdam.

[22] Kipouridis I \& Tsaklidis G, 2001, The size order of the state vector of discrete-time homogeneous Markov systems, Journal of Applied Probability, 38(2), pp. 357-368.

[23] Kulperger R \& Prakasa Rao BLS, 1989, Bootstrapping a finite state Markov chain, Sankhya: The Indian Journal of Statistics, 51(2), pp. 178-191.

[24] Lee CY \& Vairaktarakis GL, 1997, Workforce planning in mixed model assembly systems, Operations Research, 45(4), pp. 553-567.

[25] Lindgren B, 1993, Statistical theory, $4^{\text {th }}$ Edition, Chapman \& Hall, New York.

[26] MCClean S, 1991, Manpower planning models and their estimation, European Journal of Operational Research, 51(2), pp. 179-187. 
[27] MCClean S \& Abodunde T, 1978, Entropy as a measure of stability in a manpower system, Journal of the Operational Research Society, 29(9), pp. 885-889.

[28] MCClean S \& GribBin O, 1991, A non-parametric competing risks model for manpower planning, Applied Stochastic Models and Data Analysis, 7(4), pp. 327-341.

[29] McClean S, Montgomery E \& Ugwuowo F, 1998, Non-homogeneous continuous-time Markov and semi-Markov manpower models, Applied Stochastic Models and Data Analysis, 13(3), pp. 191-198.

[30] Osagiede AA \& Ekhosueni VU, 2015, Finding a continuous-time Markov chain via sparse stochastic matrices in manpower systems, Journal of the Nigerian Mathematical Society, 34(1), pp. 94-105.

[31] Osagiede AA, Enagbonma O \& Iguodala W, 2014, Outsourcing model in manpower organisations, Journal of the Nigerian Association of Mathematical Physics, 28(1), pp. 485-492.

[32] Osagiede AA, Ekhosuehi VU, Ekhosueni N \& Oyegue FO, 2012, A note on Markovian manpower models, Journal of the Nigerian Association of Mathematical Physics, 20(1), pp. 369-378.

[33] RAO PP, 1990, A dynamic programming approach to determine optimal manpower recruitment policies, Journal of the Operational Research Society, 41(10), pp. 983-988.

[34] Sethi SP, 1973, Optimal control of the Vidale-Wolfe advertising model, Operations Research, 21(4), pp. 998-1013.

[35] Stanford RE, 1980, The effects of promotion by seniority in growth-constrained organizations, Management Science, 26(7), pp. 680-693.

[36] Tyler GW, 1989, A thermodynamic model of manpower systems, Journal of the Operational Research Society, 40(2), pp. 137-139.

[37] Ugwuowo F \& McClean S, 2000, Modelling heterogeneity in a manpower system: a review, Applied Stochastic Models in Business and Industry, 16(2), pp. 99-110.

[38] Vassiliou PC, 1984, Entropy as a measure of the experience distribution in a manpower system, Journal of the Operational Research Society, 35(11), pp. 1021-1025.

[39] Vassiliou PC \& Papadopoulou A, 1992, Non-homogeneous semi-Markov systems and maintainability of the state sizes, Journal of Applied Probability, 29(1), pp. 519-534.

[40] Vassiliou PC \& Tsantas N, 1984, Maintainability of structures in nonhomogeneous Markov systems under cyclic behavior and input control, SIAM Journal on Applied Mathematics, 44(5), pp. 10141022.

[41] Verhoeven K, 1981, Corporate manpower planning, European Journal of Operational Research, 7(1), pp. 341-349.

[42] Vidale M \& Wolfe H, 1957, An operations research study of sales response to advertising, Operations Research, 5(3), pp. 370-381.

[43] WeBer TA, 2011, Optimal control theory with applications in economics, $1^{\text {st }}$ Edition, MIT Press, Cambridge.

[44] Zhu X \& Sherali HD, 2009, Two-stage workforce planning under demand fluctuations and uncertainty, Journal of the Operational Research Society, 60(1), pp. 94-103. 


\section{Appendix}

The MATLAB source code for the numerical illustration follows below.

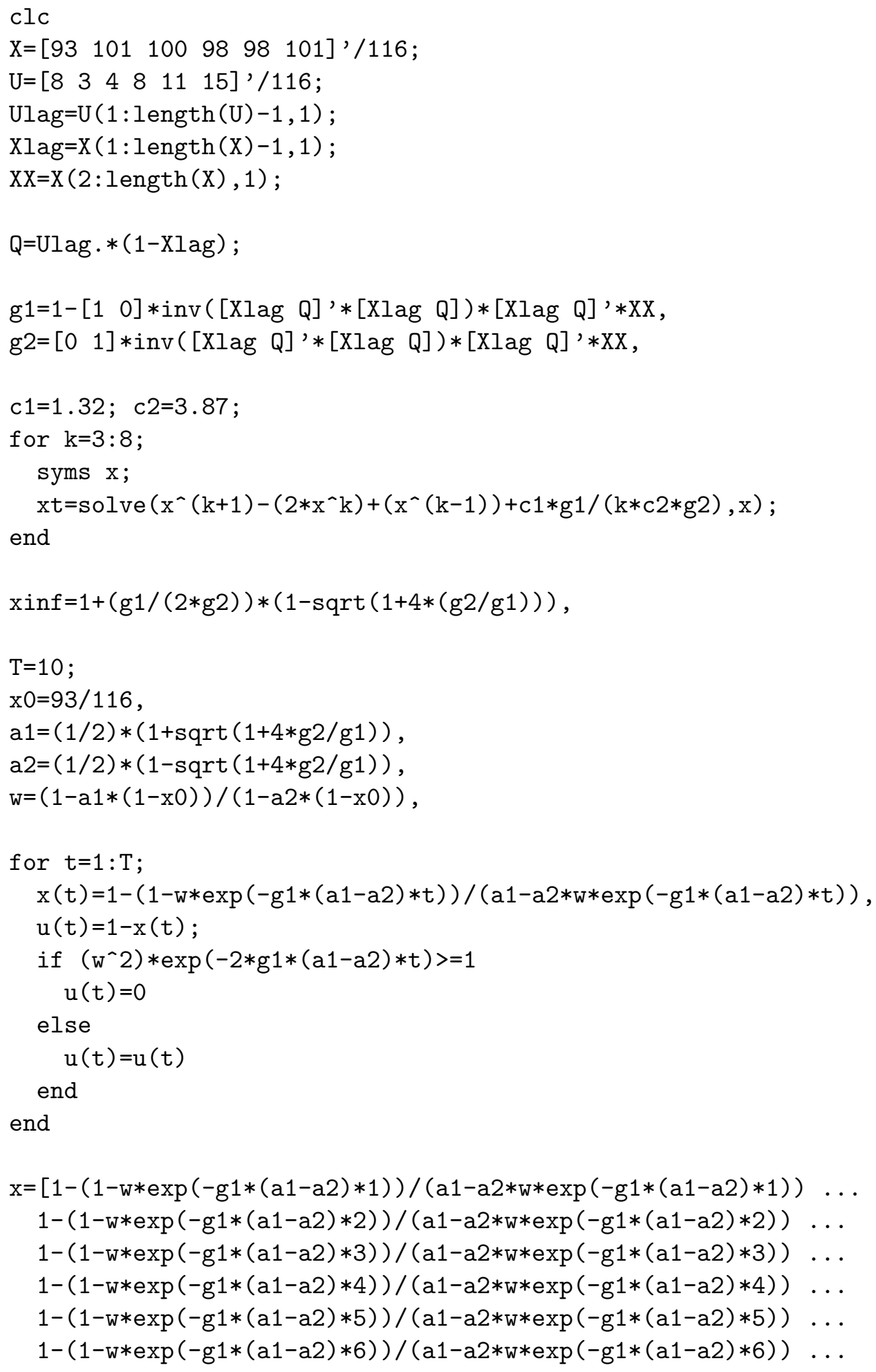




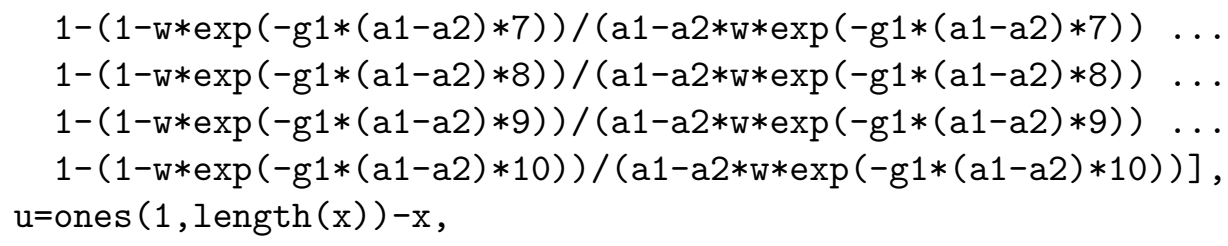

